\title{
Performance on the dementia rating scale in Parkinson's disease with dementia and dementia with Lewy bodies: comparison with progressive supranuclear palsy and Alzheimer's disease
}

\author{
D Aarsland, I Litvan, D Salmon, D Galasko, T Wentzel-Larsen, J P Larsen
}

J Neurol Neurosurg Psychiatry 2003;74:1215-1220

See end of article for authors' affiliations

.....................

Correspondence to: Dr Dag Aarsland, Section of Geriatric Psychiatry, Psychiatric Clinic, Central Hospital of Rogaland, Arm Hansen $v 20, \mathrm{~N}-4095$ Stavanger, Norway; daa@post.rps.no

Received

14 November 2002 In revised form 31 March 2003

Accepted 3 April 2003

\begin{abstract}
Background: The relation between dementia with Lewy bodies (DLB) and Parkinson's disease with dementia (PDD) is unknown.

Objectives: To compare the cognitive profiles of patients with DLB and PDD, and compare those with the performance of patients with a subcortical dementia (progressive supranuclear palsy) and a cortical dementia (Alzheimer's disease).

Design: Survey of cognitive features.

Setting: General community in Rogaland county, Norway, and a university dementia and movement disorder research centre in the USA.

Patients: 60 patients with DLB, 35 with PDD, 49 with progressive supranuclear palsy, and 29 with Alzheimer's disease, diagnosed by either standardised clinical procedures and criteria (all PDD and Alzheimer cases and $76 \%$ of cases of progressive supranuclear palsy), or necropsy (all DLB cases and $24 \%$ of cases of progressive supranuclear palsy). Level of dementia severity was matched using the total score on the dementia rating scale adjusted for age and education.

Main outcome measures: Dementia rating scale subscores corrected for age.

Results: No significant differences between the dementia rating scale subscores in the PDD and DLB groups were found in the severely demented patients; in patients with mild to moderate dementia the conceptualisation subscore was higher in PDD than in DLB ( $p=0.03)$. Compared with Alzheimer's disease, PDD and DLB had higher memory subscores $(p<0.001)$ but lower initiation and perseveration $(p=0.008$ and $p=0.021)$ and construction subscores $(p=0.009$ and $p=0.001)$. DLB patients had $a$ lower conceptualisation subscore $(p=0.004)$. Compared with progressive supranuclear palsy, PDD and DLB patients had lower memory subscores $(p<0.001)$.

Conclusions: The cognitive profiles of patients with DLB and PDD were similar, but they differed from those of patients with Alzheimer's disease and progressive supranuclear palsy. The cognitive pattern in DLB and PDD probably reflects the superimposition of subcortical deficits upon deficits typically associated with Alzheimer's disease.
\end{abstract}

$\mathrm{D}$ ementia with Lewy bodies (DLB) and Parkinson's disease with dementia (PDD) are common syndromes characterised by parkinsonism and dementia. There are few comparisons of the clinical presentations of these syndromes, and differentiation between the disorders may occasionally be difficult in clinical practice. ${ }^{12}$ Moreover, although there is some evidence suggesting pathological, ${ }^{3}$ neurochemical, ${ }^{4}$ and clinical ${ }^{5}$ similarities, the nosological relation between the two syndromes has yet to be resolved.

The cognitive impairment in DLB is characterised by marked deficits in attention and in executive, visuospatial, and constructional abilities. ${ }^{6}$ Memory is also impaired, although recall is relatively spared when compared with Alzheimer's disease. ${ }^{7}$ A similar pattern of cognitive impairment is found in Parkinson's disease. ${ }^{8}$ Nevertheless, two studies that directly compared cognition in PDD and DLB reported more executive dysfunction in the latter. ${ }^{9}{ }^{10}$ However, the mean mini-mental state examination (MMSE) ${ }^{11}$ score was only 12.5 in the DLB group compared with a nearly normal score of 24.1 in the Parkinson's disease group. ${ }^{9}$

To ensure that any between-group differences in subtest profiles are attributable to differences in the underlying nature of the two diseases rather than to differences in the global level of dementia, the two groups should be matched for overall severity of dementia. In the study by Downes et al, ${ }^{10}$ clinically diagnosed PDD and DLB cases were carefully matched for dementia severity, but the study sample was small (only 10 patients in each group), the subjects were rather young (mean ages 64.9 and 66.4 years, respectively), and the level of cognitive impairment was mild (mean verbal IQ 97.2 and 94.1, respectively). Accordingly, the representativeness of these samples can be questioned.

Cortical and subcortical pathologies occur in both DLB and PDD, with potential implications for cognitive function, but the brain structures contributing to the cognitive impairment are still not clearly identified. Furthermore, differentiation of patients with DLB or PDD from those with dementia syndromes characterised by symptoms of mainly cortical pathology, such as Alzheimer's disease, or subcortical pathology, such as progressive supranuclear palsy, may be difficult. ${ }^{1}$ Knowledge of the cognitive patterns in DLB and PDD in comparison with Alzheimer's disease and progressive supranuclear palsy could provide information about factors contributing to the cognitive

Abbreviations: DLB, dementia with Lewy bodies; MMSE, mini-mental state examination; NINDS, National Institutes of Neurological Disorders and Stroke; PDD, Parkinson's disease with dementia; SPSP, Society for Progressive Supranuclear Palsy 
dysfunction in these disorders, and could aid in the clinical diagnosis of dementia with parkinsonian syndromes and provide information on the nosological status of PDD and DLB.

With these aspects in mind, we used the dementia rating scale $^{12}$ - a brief measure of general cognitive status commonly used in both clinical practice and research-to study the performance of relatively large samples of patients with PDD, DLB, progressive supranuclear palsy, and Alzheimer's disease with similar levels of overall dementia. We hypothesised that the cognitive profiles of PDD and DLB cases are rather similar and are consistent with the presence of both cortical and subcortical dysfunction, in contrast with syndromes of cortical dementia (Alzheimer's disease) and subcortical dementia (progressive supranuclear palsy).

\section{METHODS}

Subjects

Parkinson's disease

PDD patients were recruited from a community based cohort of subjects with Parkinson's disease in the county of Rogaland, Norway. ${ }^{13}$ They were evaluated using a comprehensive battery of neurological, psychiatric, and neuropsychological tests at four year intervals for eight years. The cohort was acquired after a detailed search, and probably represents the entire Parkinson's disease population in the region (see Tandberg et al for detail $\mathrm{s}^{13}$ ). Patients who developed dementia more than one year after the onset of parkinsonism were included. In order to exclude DLB from this group, patients with dementia, repeated falls, or hallucinations at disease onset, or dementia at the primary evaluation, were not included.

A diagnosis of clinical definite, probable, or possible Parkinson's disease was made according to previously published criteria. ${ }^{14}$ In order to obtain a homogeneous population with a high diagnostic specificity for Parkinson's disease, only subjects with definite Parkinson's disease were included in the study. This required that a patient had resting tremor and at least two of the three other cardinal signs of akinesia, rigidity, and postural abnormalities. Unilateral onset and development of parkinsonism as well as a good to excellent response to a dopaminergic agent were also required.

Atypical features such as early falls, autonomic failure, or dementia, and pyramidal signs, cerebellar signs, or supranuclear gaze palsy on examination excluded the diagnosis of clinically definite Parkinson's disease. Additional exclusion criteria were other diseases that could explain the symptoms, radiological structural brain abnormalities not compatible with a diagnosis of Parkinson's disease, a history of alcohol or substance abuse in the past year, head trauma with loss of consciousness, and psychiatric disorders other than depression requiring treatment preceding the onset of the current disease.

\section{Dementia with Lewy bodies}

We included all 62 patients with necropsy confirmed DLB from the Alzheimer Research Center in San Diego (University of California San Diego) in whom the dementia rating scale had been administered during life. All these were diagnosed as having dementia at the time of their initial evaluation. They had received yearly physical, neurological, and neuropsychological assessments. Informed consent for necropsy was obtained from the next of kin immediately after the patient's death. Twenty three of the patients have been reported previously. ${ }^{15}$ The procedures that were used to prepare and study the brains of the DLB patients have been described in detail before. ${ }^{16}$ The neuropathological diagnosis of DLB required the presence of subcortical and cortical Lewy bodies. The majority of the DLB subjects also had some degree of neocortical senile plaque formation.

\section{Progressive supranuclear palsy}

The patients with progressive supranuclear palsy were 55 consecutive outpatients with this condition presenting to the
National Institutes of Neurological Disorders and Stroke (NINDS), Bethesda, Maryland, USA, for evaluation and participation in research studies, and who fulfilled the research criteria of the NINDS-SPSP (Society for Progressive Supranuclear Palsy) for the diagnosis of definite $(n=13)$, probable $(n=34)$, or possible $(n=8)$ progressive supranuclear palsy. ${ }^{17}$ Definite progressive supranuclear palsy required eventual neuropathological confirmation. The reliability and validity of the "possible" and "probable" NINDS-SPSP criteria for the diagnosis of this condition are high (as confirmed by pathology $\left.{ }^{18}\right)$. This is particularly the case for "probable" diagnosis (positive predictive value and specificity, 100\%).

\section{Alzheimer's disease}

We analysed the dementia rating scale scores from 39 consecutive patients with Alzheimer's disease attending the section of geriatric psychiatry, Rogaland Psychiatric Hospital, Stavanger, Norway. They had no clinically significant psychiatric symptoms. They were diagnosed according to ICD-10 criteria for Alzheimer's disease. ${ }^{19}$ The diagnosis was based on interviews with patients and caregivers, cognitive testing, physical examination, routine blood tests, and cranial computed tomography. None of the patients had parkinsonism.

\section{Procedures}

All patients, and when appropriate their carers, gave informed consent for participation in this research study. The dementia rating scale and the MMSE were administered according to standardised instructions. The dementia rating scale is divided into five subtests, measuring attention, initiation and perseveration, construction, conceptualisation, and memory. For individuals with Parkinson's disease, the scale has been shown to be a valid mental screening test of cognitive functioning, and the subtests show strong convergent and discriminant validity. ${ }^{20}$ To obtain the maximum number of patients with dementia, we used the most recent evaluation of the patients with Parkinson's disease. Those with motor fluctuations were assessed in the "ON" phase. The stage of parkinsonism in the patients with Parkinson's disease and progressive supranuclear palsy was rated by a neurologist using the Hoehn and Yahr scale. ${ }^{21}$ The presence of depression was determined by a composite score of 3 or more on the neuropsychiatric inventory depression item ${ }^{22}$ in Parkinson's disease, progressive supranuclear palsy, and Alzheimer's disease, and by the diagnostic interview schedule ${ }^{23}$ in the DLB group. Although the neuropsychiatric inventory is caregiver based, it significantly correlates with standard patient based rating scales of depression. ${ }^{22}$

\section{Statistical analysis}

Performance on the dementia rating scale is sensitive to age and education, but not sex. ${ }^{24}{ }^{25}$ To adjust for differences in age and education in the groups, the total score on the dementia rating scale was converted to an age and education adjusted score. $^{24}$ The adjusted scale scores range between 2 (most impaired, < 1st centile) and 18 (best possible performance, $>99$ th centile). For dementia rating scale subscores, only age corrected scores are available. As the pattern of cognitive functioning may differ for different ranges of overall cognitive function, a distinction was made between subjects with mild to moderate and severe dementia, based on the age and education corrected total score on the dementia rating scale. The cut off scores were chosen so as to obtain balanced number of patients in each category, as follows: mild to moderate dementia, a score between 4 and 7 (that is, 2nd to 18th centile range); severe dementia, a score below 4 (below the 2 nd centile).

We used $\chi^{2}$ tests for categorical variables, while betweengroup comparisons of continuous variables-including the age adjusted dementia rating scale subscores-were analysed 
Table 1 Characteristics of the patients

\begin{tabular}{|c|c|c|c|c|c|c|c|c|}
\hline & \multicolumn{4}{|c|}{ Mild to moderate dementia } & \multicolumn{4}{|c|}{ Severe dementia } \\
\hline & $\operatorname{PDD}(n=17)$ & DLB $(n=9)$ & $\operatorname{PSP}(n=20)$ & $A D(n=11)$ & $\operatorname{PDD}(n=18)$ & DLB $(n=51)$ & $\operatorname{PSP}(n=29)$ & $A D(n=18)$ \\
\hline Age at testing (years) & $77.2(5.6)$ & $73.2(7.2)$ & $66.8(5.7)^{* *}$ & $80.5(4.0)$ & $79.1(5.9)$ & $74.3(5.7)^{*}$ & $68.1(6.0)^{* *} \dagger \dagger$ & $77.9(5.1)$ \\
\hline Female (\%) & 24 & 56 & 45 & 91 * & 72 & 73 & $28 * \dagger \dagger$ & 72 \\
\hline Duration of disease (years) & $15.4(5.0)$ & $1.9(2.1)$ ** & $3.7(2.3)^{* *}$ & $2.6(1.6)^{* *}$ & $14.7(5.5)$ & $4.0(2.7)^{* *}$ & $4.8(2.3)^{* *}$ & $3.1(2.2)^{* *}$ \\
\hline Education (years) & $9.5(4.1)$ & $12.0(4.6)$ & $13.3(4.0)^{*}$ & $8.6(2.2)$ & $9.4(3.5)$ & $14.2(2.9)^{* *}$ & $14.5(2.8)^{* *}$ & $9.1(3.8) \dagger \dagger$ \\
\hline MMSE score & $23.6(2.6)$ & $23.9(2.8)$ & $28.1(2.4)^{* *} \dagger$ & $22.3(3.4)$ & $16.6(4.5)$ & $17.7(6.3)$ & $26.0(5.0)^{* *} \dagger \dagger$ & $18.2(3.6)$ \\
\hline DRS score, uncorrected & $120.6(7.0)$ & $118(10.8)$ & $125.7(5.3)$ & $125.5(3.8)$ & $87.5(23.4)$ & $95(20.7)$ & $108.6(16.4)^{*} \dagger$ & $104.6(9.4)$ \\
\hline $\begin{array}{l}\text { DRS scores, corrected for } \\
\text { age and education }\end{array}$ & $4.8(1.4)$ & $3.9(1.2)$ & $4.6(1.3)$ & $7.1(1.0)^{* *} \dagger \dagger$ & $2.1(1.0)$ & $0.9(1.0)^{\text {* * }}$ & $1.2(1.0)^{*}$ & $2.4(0.8) \dagger \dagger$ \\
\hline \multicolumn{9}{|c|}{$\begin{array}{l}\text { Values are mean (SD). } \\
\text { The diagnostic groups were compared separately in the mild and moderate-severe dementia groups using one way analysis of variance. Scheffé's post } \\
\text { hoc test was used for pairwise comparisons. Sex distribution was compared using } \chi^{2} \text { tests. } \\
\text { Different from PDD: * } p<0.05, * * p<0.001 \text {; different from } \mathrm{DLB}: \dagger p<0.05 ; \dagger p<0.001 \text {. } \\
\text { AD, Alzheimer's disease; DRS, dementia rating scale; DLB, dementia with Lewy bodies; MMSE, mini-mental state examination; PDD, Parkinson's disease } \\
\text { with dementia; PSP, progressive supranuclear palsy. }\end{array}$} \\
\hline
\end{tabular}

by one way analysis of variance (ANOVA) using SPSS version 11.0.1. The mild to moderate and severe dementia subgroups were analysed separately. If significant differences emerged, Scheffé post hoc tests were used for pairwise comparisons. Because there is a strong concentration of age adjusted memory scores at and near the lowest possible value, the Alzheimer group was excluded from these analyses for the memory scale. Memory scores for the Alzheimer group were compared with homogeneous subsets from the ANOVA analyses by exact median tests and exact $\chi^{2}$ test for the number of lowest possible scores, using the program StatXact. ${ }^{26}$

\section{RESULTS}

Thirty five cases of PDD, 60 of DLB, 49 of progressive supranuclear palsy, and 29 of Alzheimer's disease had mild to moderate or severe dementia; thus the study sample consisted of 173 subjects. The mean (SD) duration of parkinsonism preceding the onset of dementia in the PDD group was 12.3 (4.8) years. The mean Hoehn and Yahr stage was 3.5 (0.9) in the patients with Parkinson's disease and $3.8(0.7)$ in the patients with progressive supranuclear palsy (NS). Other clinical and demographic characteristics are shown in table 1. The groups differed with regard to sex, age, years of education, MMSE, and total score on the raw dementia rating scale. There were mostly small, but statistically significant, differences in the age and education corrected total scores on the dementia rating scale in the groups with mild to moderate dementia $(\mathrm{F}=14.6 ; \mathrm{df}=3,53 ; \mathrm{p}<0.001)$ and severe dementia $(\mathrm{F}=13.3 ; \mathrm{df}=3,112 ; \mathrm{p}<0.010)$.

The pairwise comparisons showed that in the mild to moderately demented cohort, the age and education corrected total score on the dementia rating scale in the Alzheimer group was significantly higher than in the PDD and DLB groups, while the other groups did not differ substantially; in the severely demented cohort, the score in the PDD group was significantly higher than in the DLB and progressive supranuclear palsy groups, and higher in the Alzheimer group than in the DLB group.

Depression was diagnosed in five patients with PDD (18\%), in nine with DLB (15\%), in nine with progressive supranuclear palsy $(18 \%)$, but in none of the Alzheimer cases $(p=0.115)$. All patients with PDD, 10 patients with progressive supranuclear palsy $(20 \%)$, and two patients with DLB $(3 \%)$ were taking antiparkinsonian agents. Psychotropic drugs with potentially adverse effects on cognition (tricyclic antidepressants, benzodiazepines, traditional antipsychotics) were being used by seven patients with PDD (20\%), six with DLB (10\%), and six with progressive supranuclear palsy ( $12 \%)$, but by none of the patients with Alzheimer's disease $(p=0.085)$. There were no significant associations between age and education adjusted total scores on the dementia rating scale and the presence or severity of depression or the use of psychotropic agents in any of the patient groups.

In the mild to moderate dementia cohort, the one way ANOVA showed highly significant between-group differences for the age adjusted dementia rating scale subscores "initiation and perseveration" ( $\mathrm{F}=6.7, \mathrm{p}=0.001)$, "construction" $(\mathrm{F}=5.5 ; \quad \mathrm{p}=0.002)$, “conceptualisation" $\quad(\mathrm{F}=6.9$; $\mathrm{p}=0.001)$, and "memory" ( $\mathrm{F}=16.3 ; \mathrm{df}=2,43 ; \mathrm{p}<0.001)$, but not for "attention" ( $\mathrm{F}=0.7, \mathrm{p}=0.57)(\mathrm{df}=3,53$ for all analyses except "memory"). In the cohort with severe dementia, the groups differed significantly on the subscores for "construction" ( $\mathrm{F}=8.9 ; \mathrm{p}<0.001)$ and "memory" ( $\mathrm{F}=32.3$; $\mathrm{df}=2,95 ; \mathrm{p}<0.01)$. There was a non-significant trend towards a difference on the "conceptualisation" subscore $(\mathrm{F}=2.5 ; \mathrm{p}=0.06)$, but there were no differences in the subscores for "attention" ( $\mathrm{F}=1.7 ; \mathrm{p}=0.12)$ and "initiation and perseveration" ( $\mathrm{F}=0.5 ; \mathrm{p}=0.7 ; \mathrm{df}=3,112$ for all analyses except "memory").

The post hoc pairwise analyses showed that in the severe dementia cohort there were no significant differences between the PDD and DLB groups, but in the mild to moderate cohort, the "conceptualisation" subscore was higher in the PDD than in the DLB group $(p=0.03)$. PDD patients had lower scores than Alzheimer's disease patients on the subscores for "initiation and perseveration" $(\mathrm{p}=0.008)$ (mild to moderate dementia) and "construction" ( $p=0.009)$ (severe dementia). DLB patients had lower scores on the subscores for "initiation and perseveration" $(\mathrm{p}=0.021)$ and "conceptualisation" $(\mathrm{p}=0.004)$ (mild to moderate dementia) and "construction" $(\mathrm{p}=0.001)$ (severe dementia) than patients with Alzheimer's disease (figs 1 and 2).

Significant differences between the PDD, DLB, and progressive supranuclear palsy groups were found on the "memory" subscore for both mild to moderate $(\mathrm{F}=117.3$; $\mathrm{df}=2,43$; $\mathrm{p}<0.0001)$ and severe dementia ( $\mathrm{F}=32.3 ; \mathrm{df}=2,95$; $\mathrm{p}<0.0001)$. As seen in figs 1 and 2 it was mainly the progressive supranuclear palsy group that differed from the others, with higher "memory" scores than the PDD and DLB groups $(\mathrm{p}<0.001)$. The "memory" scores of the PDD and DLB groups were not significantly different either for the mild to moderate or the severe dementia range. The "memory" scores were lower in the Alzheimer group than in the PDD and DLB groups with either mild to moderate dementia $(p=0.002)$ or severe dementia $(p=0.018)$. The proportion of patients with the lowest possible score (2) was significantly higher in the Alzheimer group than in the PDD group ( $p=0.0007$ for mild to moderate dementia and $\mathrm{p}=0.018$ for severe dementia). Similarly, the "memory" scores were lower in the Alzheimer group than in progressive supranuclear palsy, both in mild to moderate dementia $(p=0.0001)$ and in severe dementia 


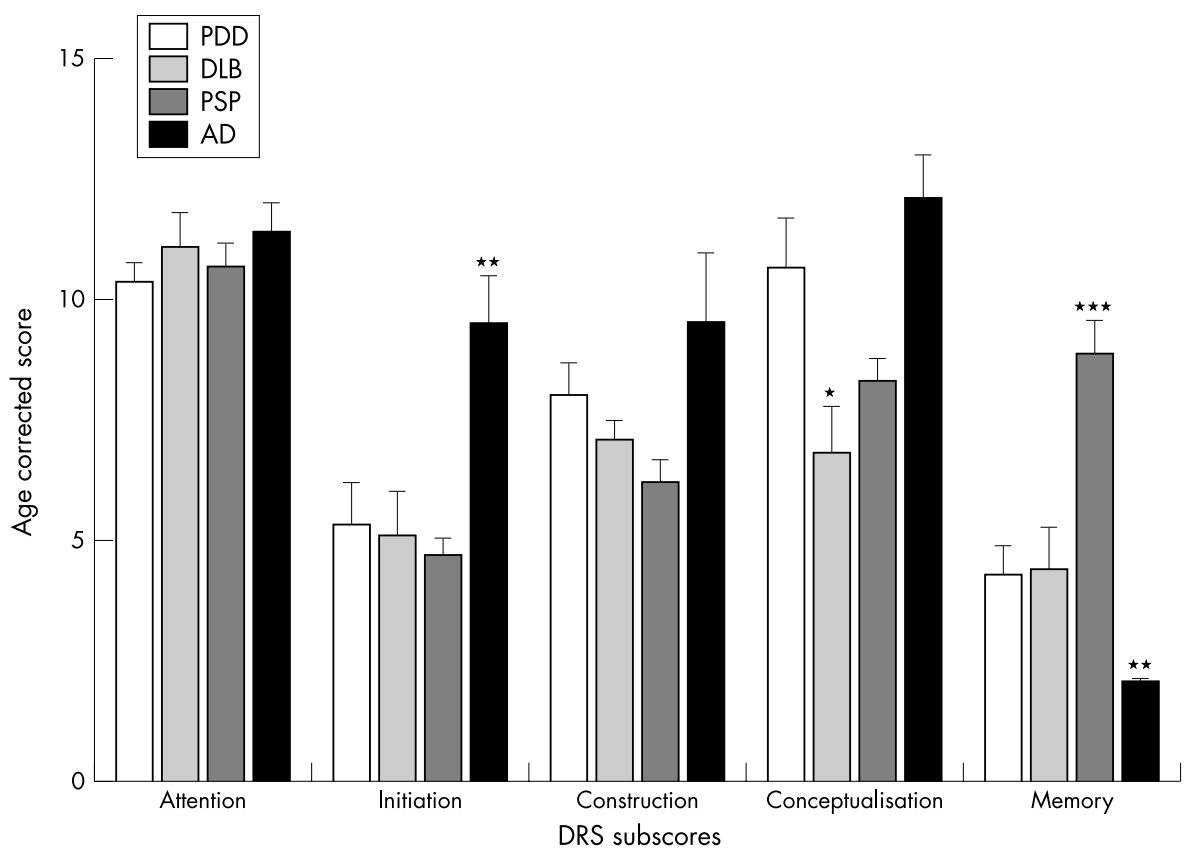

Figure 1 Dementia rating scale subscores in patients with mild to moderate dementia presented as the mean estimates and standard errors. ${ }^{*} \mathrm{p}<0.05,{ }^{* *} p<0.01,{ }^{* * *} p<0.001$. AD, Alzheimer's disease; DLB, dementia with Lewy bodies; DRS, dementia rating scale; MMSE, mini-mental state examination; PDD, Parkinson's disease with dementia; PSP, progressive supranuclear palsy.

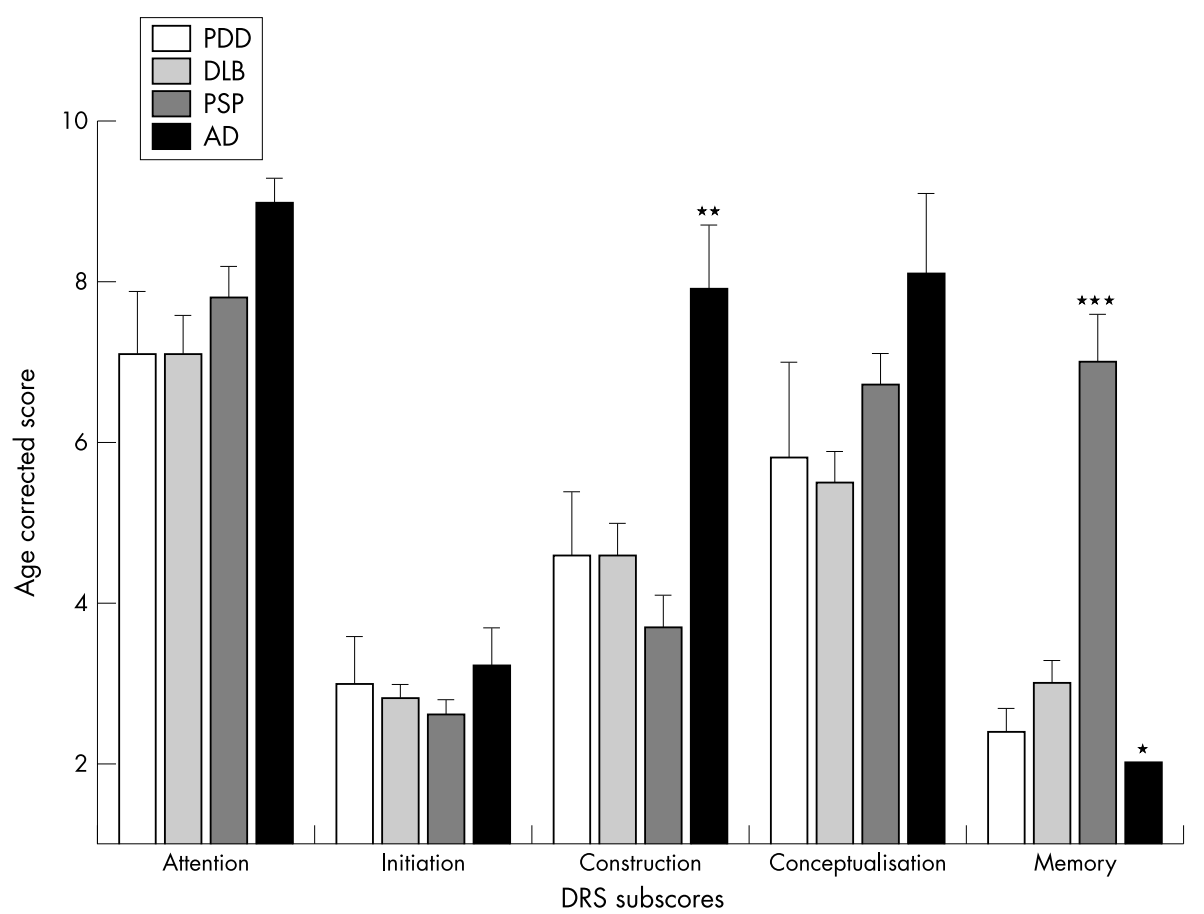

Figure 2 Dementia rating scale subscores in patients with severe dementia presented as mean estimates and standard errors. ${ }^{*} p<0.05 ;{ }^{*} p$ $<0.01 ; * *{ }^{*} p<0.001$. AD, Alzheimer's disease; DLB, dementia with Lewy bodies; DRS, dementia rating scale; MMSE, mini-mental state examination; PDD, Parkinson's disease with dementia; PSP, progressive supranuclear palsy.

$(\mathrm{p}<0.0001)$. The proportion of patients with the lowest possible score was higher in the Alzheimer group than in the group with progressive supranuclear palsy $(\mathrm{p}=0.0001$ in both severity groups).

\section{DISCUSSION}

Our main finding was a similar pattern of cognitive impairment in patients with PDD and DLB who had severe dementia, and on four of the five subscores in those with mild to moderate dementia. This finding extends recent comparative studies of motor, ${ }^{9}$ psychiatric, ${ }^{27}$ and attentional ${ }^{5}$ disturbances in PDD and DLB, highlighting the similarities between these syndromes. Given the neurochemical (for example, the cholinergic deficits ${ }^{3}$ ) and pathological (cortical Lewy bodies ${ }^{4}$ ) similarities in PDD and DLB, the clinical similarities are not surprising. The current distinction between these two conditions is based upon the timing of symptom onset. Patients are classified as having DLB when cognitive impairment or hallucinations present before or within one 
year of the onset of parkinsonism; they are classified as having PDD when the parkinsonism precedes the dementia by more than one year. ${ }^{28}$ On the basis of recent findings, PDD and DLB might better be viewed as parts of a spectrum of Lewy body disorders.

On the other hand, as both our study and a previous one ${ }^{10}$ show that executive function in patients with mild dementia and DLB is more impaired than in patients with mild PDD, it is hypothesised that the involvement of the frontal cortex occurs later in PDD. The dementia rating scale does not permit further evaluation of what leads to the memory disturbance in these two disorders, as we cannot determine whether patients fail to encode or to retrieve information. This would be necessary to establish whether the deficit results from lesions in the hippocampus or in the frontal cortex. Alternatively, it is possible that the between-group minor differences at early disease stages may reflect the heterogeneity of the underlying lesions in PDD and DLB (Lewy bodies, amyloid plaques, parkinsonism associated with other disorders, vascular disease).

We found that the cognitive pattern of patients with DLB and PDD differed from that of patients with cortical dementia (Alzheimer's disease) and subcortical dementia (progressive supranuclear palsy). On the memory subscale of the dementia rating scale-which is related to hippocampal atrophy ${ }^{29}$ - the DLB and PDD subjects were more impaired than the patients with progressive supranuclear palsy, and less impaired than the patients with Alzheimer's disease. These findings are consistent with previous reports. ${ }^{75}{ }^{30-32}$ Hippocampal atrophy is found in Parkinson's disease ${ }^{33}$ and $\mathrm{DLB}^{34}$ and is more pronounced in DLB than in progressive supranuclear palsy. ${ }^{34}$ The "initiation and perseveration" subscore, related to frontal atrophy, and the "construction" score, related to bilateral temporal atrophy, ${ }^{29}$ were less impaired in Alzheimer's disease than in PDD and DLB, consistent with previous studies. ${ }^{15}{ }^{30}$ The cognitive pattern in patients with DLB and PDD may be characterised as reflecting the superimposition of frontosubcortical cognitive deficits upon the cognitive deficits typically associated with Alzheimer's disease.

A confounding factor in this study was that diagnostic group was segregated by research centre. Thus the findings may have been influenced by different approaches to diagnosis or evaluation. However, the clinical diagnoses were based on standardised procedures and criteria, and the diagnosis of all DLB patients and of at least $85 \%$ of patients with progressive supranuclear palsy was either confirmed by necropsy using standardised criteria (definite) or met the definite clinical criteria (100\% accuracy). The PDD patients were followed longitudinally by the same neurologists for at least four years, a procedure that has been shown to yield a highly accurate clinical diagnosis. ${ }^{35}$

The clinical presentation of PDD and DLB patients overlaps. Thus care was taken to ensure a high specificity of diagnosis in both groups. All DLB patients were demented at their first evaluation, and the clinical diagnosis was confirmed by necropsy in every case. All PDD patients were cognitively intact and without hallucinations at their first evaluation, and the mean duration of Parkinson's disease before dementia began was 12.4 years. The DLB and PDD groups were therefore distinguished clinically according to current criteria. ${ }^{28}$

It could be argued that the dementia rating scale may not be sensitive or comprehensive enough to detect possible differences between patients with DLB and PDD. For example, the construction subscale has been shown to have less than optimal discriminant validity ${ }^{20}$; however, the other four subscales of the dementia rating scale have strong convergent and discriminant validity. ${ }^{36}$ They account for more of the variability in the performance on a comprehensive battery of tests assessing a wide range of cognitive functions in Parkinson's disease patients than the MMSE or a selection of neuropsychological tests known to be sensitive to specific cognitive deficits in Parkinson's disease..$^{20}$ Previous studies, ${ }^{150} 37$ as well as our results, show that the dementia rating scale can differentiate between different types of dementia, suggesting that this is in fact a sensitive and valid measure of impairment of different cognitive domains. As the dementia rating scale measures executive functions, it is more sensitive to frontal dysfunction-the main cognitive problem in patients with progressive supranuclear palsy-whereas the MMSE is relatively insensitive to frontal dysfunction. This is shown by the higher MMSE scores in patients with progressive supranuclear palsy, suggesting that the dementia rating scale is useful for assessing cognition in these patients. Nevertheless, it is possible that detailed neuropsychological testing would have identified more widespread differences between the DLB and PDD patients. This issue needs further investigation.

Furthermore, our groups differed with regard to sample selection. The PDD patients were drawn from an epidemiological population of cases of Parkinson's disease and therefore represent the full spectrum of disease severity and presentation. The DLB and progressive supranuclear palsy groups, on the other hand, were referrals to university dementia or movement disorder research centres, introducing a potential bias towards the inclusion of more severely affected or complicated cases and the exclusion of mildly affected cases. The groups differed with regard to age, sex, and years of education, and their performance on the dementia rating scale is influenced by age and the level of education, but not by sex. ${ }^{24}$ To control for potential bias based on demographic differences, the overall level of dementia was based on age and education corrected norms, and the dementia rating scale subscores were converted to age corrected scores. An education based differential influence on the dementia rating scale performance cannot be entirely excluded, however.

The strengths of our study include the relatively large samples, the prospective design that aided in the diagnosis of PDD, and the necropsy confirmation in DLB-the most difficult of the syndromes to diagnose clinically-and in $24 \%$ of the patients with progressive supranuclear palsy. The DLB and progressive supranuclear palsy groups are among the largest cohorts yet reported, and the PDD group was community based. Thus the patient groups may be representative of patients with these brain diseases. The overall severity of dementia was comparable in the four diagnostic groups. However, there were small between-group differences, particular in patients with severe dementia. It is possible that these differences may have influenced the pattern of cognition differentially in the four groups.

\section{Conclusions}

A similar pattern of cognitive impairment was found in patients with PDD and DLB with severe dementia, and was different from that in patients with cortical and subcortical dementia. Our findings suggest but do not prove that the underlying mechanisms of cognitive decline in Parkinson's disease are similar to those in DLB, but different from those in Alzheimer's disease and progressive supranuclear palsy.

Authors' affiliations

D Aarsland, Section of Geriatric Psychiatry, Psychiatric Clinic, Central Hospital of Rogaland, Stavanger, and School of Medicine, University of Bergen, Norway

I Litvan, Department of Neurology, University of Louisville, Louisville, Kentucky, USA

D Galasko, Department of Neurosciences, University of California, San Diego, California, USA

T Wentzel-Larsen, Centre for Clinical Research, Haukeland University Hospital, Bergen, Norway

J P Larsen, Department of Neurology, Central Hospital of Rogaland,

Stavanger, and School of Medicine, Úniversity of Bergen, Bergen,

Norway

Competing interests: none declared 


\section{REFERENCES}

1 Litvan I, Maclntyre A, Goetz CG, et al. Accuracy of the clinical diagnoses of Lewy body disease, Parkinson's disease, and dementia with Lewy bodies. Arch Neurol 1998;55:969-78.

2 Lopez OL, Becker JT, Kaufer DI, et al. Research evaluation and prospective diagnosis of dementia with Lewy bodies. Arch Neurol 2002:59:43-6.

3 Tiraboschi $\mathbf{P}$, Hansen LA, Alford $M$, et al. Cholinergic dysfunction in diseases with Lewy bodies. Neurology 2000;54:407-1 1 .

4 Harding AJ, Broe GA, Halliday GM. Visual hallucinations in Lewy body disease relate to Lewy bodies in the temporal lobe. Brain 2002; 125:391-403.

5 Ballard C, Aarsland D, McKeith IG, et al. Fluctuations in attention. PD dementia vs DLB with parkinsonism. Neurology 2002;59:1714-20.

6 Galasko D, Salmon DP. The Alzheimer-Parkinson disease connection. In: Clark CM, Trojanowski JQ. Neurodegenerative dementias. New York: McGraw-Hill, 2000:229-40.

7 Ballard CG Ayre G, O'Brien J, et al Simple standardized neuropsychological assessments aid in the differential diagnosis of dementia with Lewy bodies from Alzheimer's disease and vascular dementia. Dement Geriatr Cogn Disord 1999;10:104-8.

8 Dubois B, Pillon B. Cognitive deficits in Parkinson's disease. J Neurol 1997;244:2-8

9 Gnanalingham KK, Byrne EJ, Thornton A, et al. Motor and cognitive function in Lewy body dementia: comparison with Alzheimer's and Parkinson's diseases. J Neurol Neurosurg Psychiatry 1997;62:243-52.

10 Downes JJ, Priestly NM, Doran M, et al. Intellectual, mnemonic, and frontal functions in dementia with Lewy bodies: a comparison with early and advanced Parkinson's disease. Behav Neurol 1998/1999;11:17383.

11 Folstein MF, Folstein SE, McHugh PR. "Mini-mental state": a practical method for grading the mental state of patients for the clinician. J Psychiatr Res 1975;12:189-98.

12 Mattis S. Dementia rating scale. In: Bellak L, Karasu TB, eds. Geriatric psychiatry. A handbook for psychiatrists and primary care physicians. New York: Grune and Stratton, 1976:108-21.

13 Tandberg E, Larsen JP, Nessler EG, et al. The epidemiology of Parkinson' disease in the county of Rogaland, Norway. Mov Disord 1995; 10:541-9.

14 Larsen JP, Dupont E, Tandberg E. The clinical diagnosis of Parkinson's disease: proposal of diagnostic subgroups classified at different levels of confidence. Acta Neurol Scand 1994;84:242-51.

15 Connor DJ, Salmon DP, Sandy TJ, et al. Cognitive profiles of autopsy-confirmed Lewy body variant vs pure Alzheimer's disease. Arch Neurol 1998;55:994-1000.

16 Hansen LA, Samuel W. Criteria for Alzheimer's disease and the nosology of dementia with Lewy bodies. Neurology 1997;48:126-32.

17 Litvan I, Agid Y, Calne D, et al. Clinical research criteria for the diagnosis of progressive supranuclear palsy (Steele-RichardsonOlszewski syndrome): report of the NINDS-SPSP International Workshop. Neurology 1996;47: 1-9.

18 Lopez OL, Litvan I, Catt KE, et al. Reliability and validity of four clinical diagnostic criteria for the diagnosis of neurodegenerative dementias. Neurology 1999;53:1292-9.
19 World Health Organisation. International classification of diseases, 10th ed. Geneva: WHO, 1992.

20 Brown GG, Armstrong Rahill A, Gorell JM, et al. Validity of the dementia rating scale in assessing cognitive function in Parkinson's disease. $J$ Geriatr Psychiatry Neurol 1999;12:180-8.

21 Hoehn MH, Yahr MD. Parkinsonism: onset, progression, and mortality. Neurology 1967; 17:427-42.

22 Cummings JL, Mega M, Gray K, et al. The Neuropsychiatric Inventory: comprehensive assessment of psychopathology in dementia. Neurology 1994:44:2308-14.

23 Robins LN, Heltzer JE, Croughan J, et al. National Institute of Mental Health Diagnostic Interview Schedule: its history, characteristics, and validity. Arch Gen Psychiatry 1981;38:381-9.

24 Lucas JA, Ivnik RJ, Smith GE, et al. Normative data for the Mattis dementia rating scale. J Clin Exp Neuropsychol 1998, 20:536-47.

25 Schmidt R, Freidl W, Fazekas F, et al. The Mattis dementia rating scale: normative data from 1001 healthy volunteers. Neurology 1994;44:964-6.

26 StatXact 5. User manual. Cambridge, MA: Cytel Software Corporation, 1989-2001.

27 Aarsland D, Ballard C, Larsen JP, et al. A comparative study of psychiatric symptoms in dementia with Lewy bodies and Parkinson's disease with and without dementia. Int J Geriatr Psychiatry $2001 ; 16: 528-36$.

28 McKeith IG, Galasko D, Kosaka K, et al. Consensus guidelines for the clinical diagnosis of dementia with Lewy bodies (DLB): report of the consortium on DLB international workshop. Neurology 1996;47: $1113-24$

29 Fama R, Sullivan EV, Shear $P$, et al. Selective cortical and hippocampal volume correlates of Mattis Dementia Rating Scale in Alzheimer's disease. Arch Neurol 1997;54:719-28.

30 Paolo AM, Troster Al, Glatt SL, et al. Differentiation of the dementias of Alzheimer's and Parkinson's disease with the dementia rating scale. J Geriatr Psychiatry Neurol 1995:8:184-8.

31 Litvan I, Mohr E, Williams J, et al. Differential memory and executive functions in demented patients with Parkinson's disease and Alzheimer's disease. J Neurol Neurosurg Psychiatry 1991;54:25-9.

32 Pillon B, Dubois B, Ploska A, et al. Severity and specificity of cognitive impairment in Alzheimer's, Huntington's, and Parkinson's diseases and progressive supranuclear palsy. Neurology 1991;41:634-43.

33 Riekkinen $\mathbf{P}$, Kejonen K, Laakso MP, et al. Hippocampal atrophy is related to impaired memory, but not frontal functions in non-demented Parkinson's disease patients. NeuroReport 1998:9:1507-1 1.

34 Cordato NJ, Halliday GM, Harding AJ, et al. Regional brain atrophy in progressive supranuclear palsy and Lewy body disease. Ann Neurol 2000;47:718-28.

35 Hurtig HI, Trojanowski JQ, Galvin J, et al. Alpha-synuclein cortical Lewy bodies correlate with dementia in Parkinson's disease. Neurology 2000;54:1916-21.

36 Hofer SM, Piccinin A. Analysis of structure and discriminative power of the Mattis dementia rating scale. J Clin Psychol 1996;52:395-409.

37 Salmon DP, Kwo-on-Yuen PF, Heindel WC, et al. Differentiation of Alzheimer's disease and Huntington's disease with the dementia rating scale. Arch Neurol 1989;46:1204-8. 